\title{
Research Paper: The Effects of Quetiapine on Craving and Withdrawal Symptoms in Methamphetamine Abuse: A Randomized, Double-Blind, Placebo-Controlled Trial
}

\author{
Najme Sadat Javdan ${ }^{1}$ (D), Amir Ghaderi ${ }^{1,2}$ (D), Hamid Reza Banafshe ${ }^{1,3,4^{*}}$ (D) \\ 1. Department of Addiction Studies, Faculty of Medicine, Kashan University of Medical Sciences, Kashan, Iran. \\ 2. Clinical Research Development Unit Matini / Kargarnejad Hospital, Kashan University of Medical Sciences, Kashan, Iran. \\ 3. Department of Pharmacology, Faculty of Medicine, Kashan University of Medical Sciences, Kashan, Iran. \\ 4. Research Center for Physiology Science, Kashan University of Medical Sciences, Kashan, Iran.
}

$\begin{aligned} & \text { Use yourdevice to scan } \\ & \text { and read the article online }\end{aligned}$
Methamphetamine Abuse: A Randomized, Double-Blind, Placebo-Controlled Trial. International Journal of Medical Toxicology
and Forensic Medicine. 2020; 10(4):29374. https://doi.org/10.32598/ijmtfm.v10i4.29374

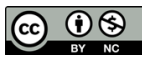

Article info:

Received: 10 Mar 2020

First Revision: 16 Mar 2020

Accepted: $15 \mathrm{Jul} 2020$

Published: 06 Dec 2020

\section{Keywords:}

Methamphetamine, Quetiapine, Craving, Withdrawal syndrome

\section{ABSTRACT}

Background: Patients with Methamphetamine Abuse (MA) are susceptible to many complications like craving, and withdrawal symptoms. These trials were designed to evaluate the effect of quetiapine administration on craving and withdrawal symptoms in MA abuse.

Methods: This trial was conducted on 60 people with MA abuse to receive either 100 mg quetiapine $(n=30)$, or placebo $(n=30)$ every day for 2 months. The Desire for Drug Questionnaire (DDQ) and Amphetamine Withdrawal Questionnaire (AWQ) scores were evaluated at baseline and after 2 months' intervention. For data analysis, $t$ test, and the Chisquare test were applied in SPSS v. 18.

Results: Quetiapine significantly decreased DDQ $(\mathrm{P}=0.002)$ and AWQ symptoms $(\mathrm{P}=0.001)$ compared to the placebo. Furthermore, there was a significant difference among groups in terms of the frequency of negative urine tests $(\mathrm{P}<0.001)$.

Conclusion: This trial showed that administration of quetiapine supplements for 2 months in individuals with MA abuse had beneficial effects on craving and withdrawal syndrome.

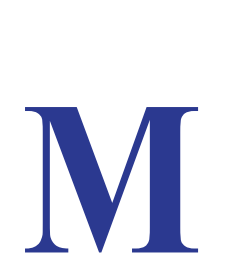

\section{Introduction:}

ethamphetamine (MA) abuse is one of the crucial health problems in the world [1]. For the first time, in 2005, MA and its effects had been reported from Southeast Asia [2]. MA has been found an important health problem and the MA depen- dence is approximately $1 \%$ among Iranian [2-4]. New insights into this condition indicated that a variety of actions could be related to reducing MA abuse. Methadone Maintenance Treatment (MMT) programs can be introduced as an effective action which enables to enhance the quality of life or social functioning [5]. Despite several implementations of the MMT, some challenges, and barriers have remained. Many evidence reports re-

* Corresponding Author:

Hamid Reza Banafshe, PhD.

Address: Department of Pharmacology, Faculty of Medicine, Kashan University of Medical Sciences, Kashan, Iran.

Tel: +98 (31) 55463378

E-mail: banafshe57@hotmail.com 
vealed that the prevalence of MA is increasing in MMT programs $[3,6,7]$. MA abuses are linked to many psychiatric impacts (e.g., increase craving, and withdrawal syndrome) $[8,9]$.

Pharmacotherapy has emerged as a new venue in the reduction of substance abuse. Despite the many benefits of these therapies, there are different challenges for the utilization of pharmacotherapy agents in the treatment of drug abuse. The pharmacological impact of antipsychotic agents for drug abuse therapy has been documented in psychotic disorder subjects [10]. Quetiapine is a pharmacological agent which is known as an atypical antipsychotic drug. It could be employed for treating different disorders such as cognitive disturbance in chronic schizophrenia [11]. Much evidence documented that quetiapine could be used for decreasing alcohol craving and withdrawal symptoms [10].

Previous reports indicated that quetiapine is associated with the ameliorated impacts on clinical symptoms. Current evidence indicated that quetiapine administration at a dosage of $100 \mathrm{mg} / \mathrm{d}$ for 2 months ameliorate psychiatric symptoms e.g., cognitive and mental health disorder [12]. Sattar et al. [10] demonstrated that using an average of $153 \mathrm{mg} / \mathrm{d}$ quetiapine for 1 month in subjects with coexisting substance abuse disturbance show favorable impacts on the craving scale. Furthermore, some evidence revealed that administration of quetiapine dosage of $50-100 \mathrm{mg} / \mathrm{d}$, for 3 months to individuals with cocaine dependence, and bipolar disorder show pharmacological impacts on cocaine cravings [13].

Quetiapine could decrease the dopamine blockade, which may result in a reduction in substance abuse. Moreover, quetiapine enables to inhibits the dopamine receptors in the limbic system, which be associated with minimal blockade of dopamine in the reward process $[10,11]$. To the best of our knowledge, the evidence on the impacts of quetiapine on clinical symptoms in MA abuses is little. So, we aimed in this trial to evaluate the effects of quetiapine on craving and withdrawal symptoms in MA abuse.

\section{Materials and Methods}

\section{Study design}

This randomized, double-blinded, placebo-controlled trial was registered on the Iranian website (http://www. irct.ir: IRCT20171106037290N1), the Primary Registry in the World Health Organization (WHO) Registry Network set up in collaboration with the Ministry of Medi- cal and Health Education. This trial was conducted on 60 MA abusers under MMT, aged 18-65 years who were referred to the Golabchi Clinic (KAUMS clinic center). The study protocol was approved by the Research Ethics Committee of Kashan University of Medical Sciences (KAUMS). All informed consent forms were reviewed by the Research Ethics Committee of KAUMS. The inclusion criteria were aged 18 to 65 years, under MMT, MA abuser, seeking treatment for MA abuse, as assessed by DSM-IV, at least weekly MA use during a preceding three months. Also, the exclusion criteria were psychotic symptoms, suicide, cocaine, and alcohol dependence, cardiovascular disturbance, recent use of over-the-counter or prescription drugs that would be expected to have major interaction with quetiapine, metabolic diseases (e.g., epilepsy, diabetes, and neurological).

\section{Study protocol}

Patients were randomized into two groups and supplemented with either $100 \mathrm{mg}$ quetiapine or placebo $(\mathrm{n}=30)$, every day for 2 months. We have used the mentioned dose of quetiapine based on a past study in individuals with liver diseases, bipolar disorder, and cocaine dependence $[13,14]$. All individuals completed three physical activity and 3-day food records as metabolic equivalents [15] at weeks $0,2,4,6$, and 8 of the treatment. Quetiapine and placebo tablets were prepared in the same way. They had an identical shape, color, size, odor, and texture. The tablets were packed in the same container with a code number. The tablets were prepared by a pharmacist of Zahravi, Tabriz City, Iran. Thus, participators, physician, and other investigators were all blind to the treatment group assignments. The randomization was conducted using computer-generated random numbers. Allocation and randomization were concealed from the patients and researchers until the completion of the final analyses.

\section{Study measurements}

\section{Clinical measures}

Craving: The original Desire for Drug Questionnaires (DDQ) such as 13 questions for three main craving components, desire, and intention to drug use, negative reinforcement, and control. High validity and reliability of the DDQ have been reported for the evaluation of periodic and instant cravings in Iran. The DDQ has a good level of internal consistency (Cronbach alpha $=0.89)[16]$. 


\section{Withdrawal symptoms}

The Amphetamine Withdrawal Questionnaire (AWQ) is a short, valid, and reliable measure for assessing amphetamine withdrawal scales. The items were rated as follows: 1. Not at all; 2. Very little; 3. A little; 4. Quite a lot; 5. Very much [17]. The possible range of scores is 0-40 with a higher score indicating greater severity. The high validity of the AWQ for questions individually and the whole questionnaire was $80 \%$ and $86.5 \%$. The internal consistency (Cronbach alpha) was 0.84 for the whole questionnaire [18].

\section{Sample size}

To evaluate the sample size, we used a clinical trial sample size formula where type I $(\alpha)$, and type II errors $(\beta)$ were 0.05 and 0.20 (power $=80 \%$ ), respectively. Based on a prior study [19], we used a Standard Deviation (SD) of 8.9 and a change in the mean of 5.1 days, considering DDQ as the main variable. The calculation showed that 25 patients should have been enrolled in each group. Assuming a dropout of $20 \%$ patients in each group, the final sample size was determined to be 30 patients per each group.

\section{Statistical analysis}

To test the normality of variables, the KolmogorovSmirnov test was used. Dietary characteristics, an-

Table 1. General characteristics of the study participants $(n=28)$

\begin{tabular}{|c|c|c|c|}
\hline \multirow{2}{*}{ Variable } & \multicolumn{2}{|c|}{ No. (\%) / Mean \pm SD } & \multirow{2}{*}{ P2 } \\
\hline & Placebo Group & Quetiapine Group & \\
\hline Age $(y)$ & $38.7 \pm 10.0$ & $37.1 \pm 9.0$ & 0.68 \\
\hline Height (cm) & $169.2 \pm 7.5$ & $170.6 \pm 6.5$ & 0.14 \\
\hline Weight at study baseline (kg) & $74.8 \pm 11.9$ & $74.4 \pm 11.7$ & 0.38 \\
\hline Weight at the end-of-trial (kg) & $72.1 \pm 10.5$ & $72.3 \pm 9.2$ & 0.46 \\
\hline Weight change (kg) & $-0.39 \pm 2.1$ & $0.14 \pm 2.5$ & 0.40 \\
\hline $\mathrm{BMI}$ at study baseline $\left(\mathrm{kg} / \mathrm{m}^{2}\right)$ & $26.2 \pm 4.2$ & $24.8 \pm 3.5$ & 0.18 \\
\hline $\mathrm{BMI}$ at the end-of-trial $\left(\mathrm{kg} / \mathrm{m}^{2}\right)$ & $26.0 \pm 4.2$ & $24.8 \pm 3.1$ & 0.23 \\
\hline BMI change $\left(\mathrm{kg} / \mathrm{m}^{2}\right)$ & $-0.13 \pm 0.7$ & $0.05 \pm 0.8$ & 0.37 \\
\hline \multicolumn{4}{|l|}{ Use of other drugs (\%) } \\
\hline None & $23(82.2)$ & $19(67.8)$ & \\
\hline Sedative-Hypnotic & $2(7.1)$ & $5(17.9)$ & $0.40^{+}$ \\
\hline Antidepressants & $3(10.7)$ & $4(14.3)$ & \\
\hline Age of the first MA experience & $32 \pm 7.8$ & $32.6 \pm 8.6$ & 0.77 \\
\hline Dose of MA use (g/wk) & $0.59 \pm 0.4$ & $0.47 \pm 0.4$ & 0.29 \\
\hline Frequency of MA use (wk) & $3.2 \pm 1.4$ & $2.7 \pm 1.4$ & 0.17 \\
\hline Duration of MA use (y) & $2.2 \pm 1.0$ & $2.5 \pm 1.1$ & 0.33 \\
\hline Methadone dose $(\mathrm{mL} / \mathrm{d})$ & $17.3 \pm 4.4$ & $18.9 \pm 5.4$ & 0.23 \\
\hline Duration of MMT (y) & $5.5 \pm 2.0$ & $6.1 \pm 2.1$ & 0.28 \\
\hline
\end{tabular}

Obtained from the independent $\mathrm{t}$ test;

† Obtained from the Pearson Chi-square tes;

MA: Methamphetamine; MMT: Methadone Maintenance Treatment; BMI: Body Mass Index. 
Table 2. The effect of Quetiapine on craving and withdrawal symptoms in methadone maintenance treatment patients $(n=28)$

\begin{tabular}{|c|c|c|c|c|c|c|c|}
\hline \multirow{3}{*}{ Variables } & \multicolumn{6}{|c|}{ Mean \pm SD } & \multirow{3}{*}{$\mathbf{P 2}$} \\
\hline & \multicolumn{3}{|c|}{ Placebo Group } & \multicolumn{3}{|c|}{ Quetiapine Group } & \\
\hline & Baseline & Week 8 & Change & Baseline & Week 8 & Change & \\
\hline $\mathrm{DDQ}$ & $41.6 \pm 8.5$ & $40.7 \pm 8.3$ & $-0.89 \pm 3.4$ & $40.5 \pm 8.6$ & $35.3 \pm 7.1$ & $-5.14 \pm 6.8$ & 0.002 \\
\hline AWQ & $36.3 \pm 6.9$ & $37.0 \pm 6.3$ & $0.67 \pm 2.7$ & $35.9 \pm 6.1$ & $33.0 \pm 6.0$ & $-2.96 \pm 4.0$ & 0.001 \\
\hline
\end{tabular}

2. Obtained from the independent $\mathrm{t}$ test.

AWQ: Amphetamine Withdrawal Questionnaire; DDQ: Desire for Drug Questionnaire.

thropometric, study outcomes, and of the intervention groups were compared using the independent sample $t$ test. Also, qualitative data were analyzed using the Chisquare test. The data were presented as the mean differences with $95 \%$ confidence intervals. The $\mathrm{P}$ values of less than 0.05 were considered statistically significant. All statistical analyses were done with SPSS version 18 .

\section{Results}

Two participants in the supplemented group and 2 in the placebo group left the study for personal reasons (Figure 1). No serious side effects were reported following the consumption of quetiapine in MA abuse patients under MMT. Though grade 1 side effects were reported in 7 patients in the quetiapine group (daytime sleepiness $(n=3)$, headache $(n=3)$, and dizziness $(n=1))$, nobody was excluded from the trial.

There was no significant difference among the two groups in terms of height, weight, age, BMI, frequency of MA use, duration of MA use, the dose of MA use, age of MA experience, duration of methadone use, and methadone dose (Table 1).

Quetiapine was significantly decreased DDQ score $(\mathrm{P}=0.002)$ and AWQ score $(\mathrm{P}=0.001)$ (Table 2). Besides, there was a significant difference between the two groups in terms of the frequency of negative urine tests in the treatment group $(\mathrm{P}<0.001)$. Also, in this study, $\mathrm{B}=1.28$ (e $\mathrm{B}=\mathrm{e} 1.28=3.5$ ). In other words, patients in the treatment group are 3.5 times more likely to have a negative urine test than the control group (Figure 2). Changes in DDQ

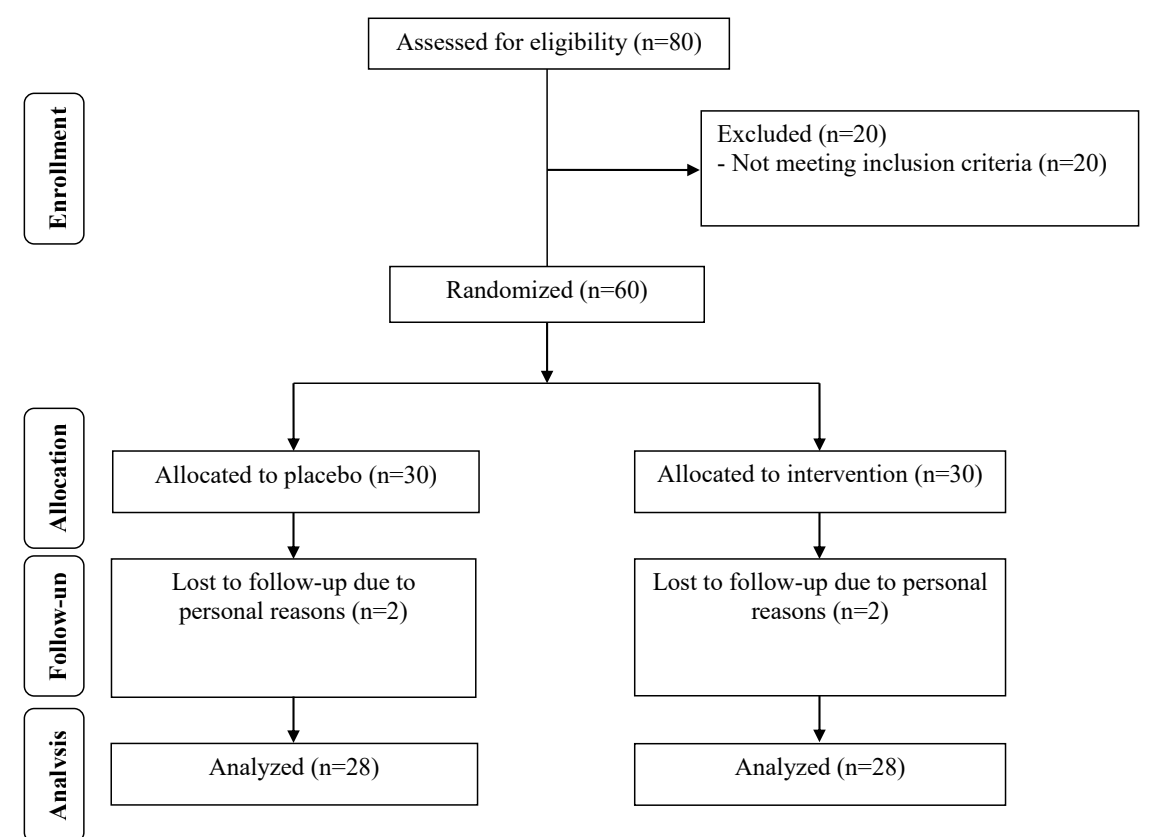

Figure 1. Summary of study patients' selection 
and AWQ score in patients MA abuse under MMT receiving quetiapine and placebo are presented in Figure 3.

\section{Discussion}

In our study, we observed the effects of quetiapine intake on craving and withdrawal symptoms after 8 weeks of supplementation in cases with MA abuse. Our report indicated that receiving quetiapine for 2 months by subjects with MA abuse who were under MMT, in comparison to the control group, decreased craving and withdrawal signs. For the first time, this report assessed the impacts of quetiapine on craving and withdrawal signs of subjects with MA abuse who were under MMT.

MA abuse is related to various problems such as craving and withdrawal syndrome [8, 20]. Many studies proposed the benefits of new antipsychotic drugs in decreasing drug abuse-related disorders in cases with a dual diagnosis [21, 22]. In a study, Brown et al. [13] revealed that administration of quetiapine could significantly enhance the psychiatric signs and cravings dosage of $50-100 \mathrm{mg} / \mathrm{d}$ for 3 months. Another reports, showed that administration of 50-300 $\mathrm{mg}$ /day quetiapine (average $153 \mathrm{mg} /$ day) for 28 day to in patients with substance dependence disorders was beneficial for the treatment of craving scale and withdrawal symptoms [10].

Rizkallah et al. [19] observed that administration of quetiapine was related to a better craving score. Moreover, various reports proposed an anti-craving impact

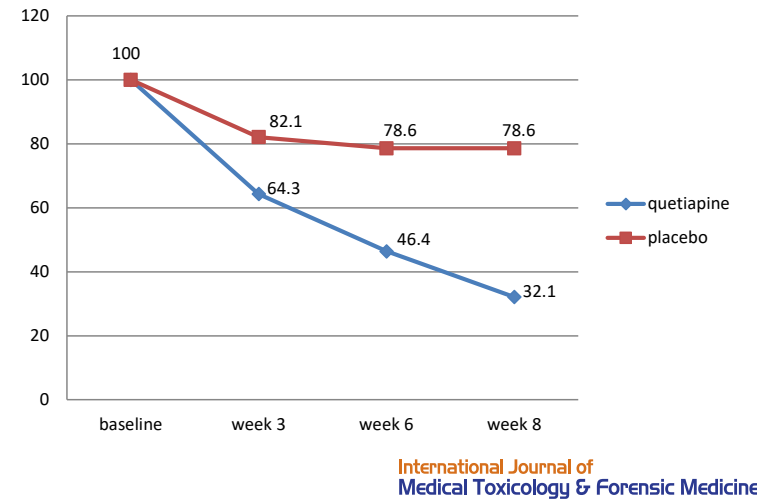

Figure 2. The effect of quetiapine on urinary test methamphetamine

of quetiapine in psycho-stimulants and alcohol [23, 24]. Although, the high dopamine receptor binding of antipsychotics could be related to the aggravation in craving in the subjects with methamphetamine abuse [25, 26]. Some reasons for discrepant results may be various study designs, lack of observing baseline values of dependent variables as well as characteristics of study individuals, various dosages of quetiapine administration also the duration of the study. It is well found that quetiapine exerts its addictive effects via the limbic system's reward process, possibly mediated by the release of the neurotransmitter dopamine [27].

Pharmacological agents that block dopamine could inhibit this process, possibly resulting in attempts to counter this blockade via elevating MA abuse. This could be
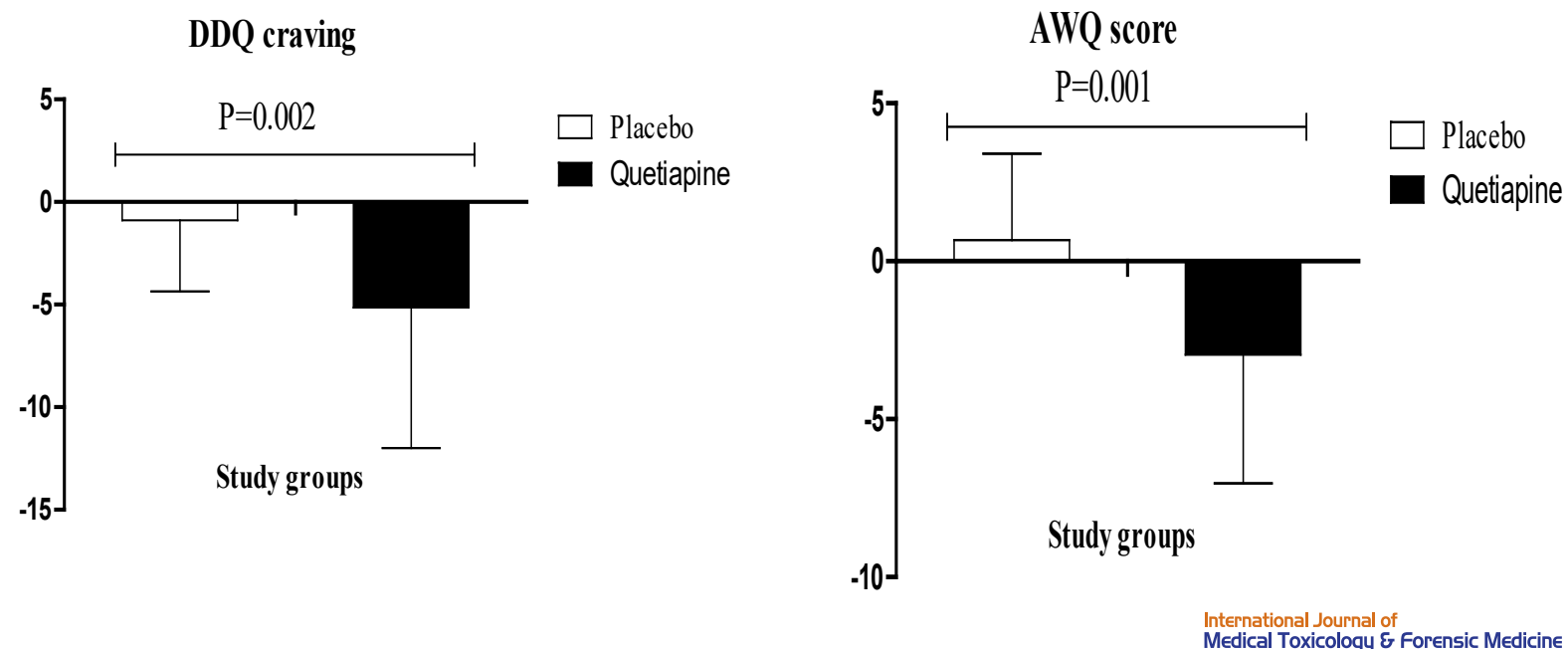

Figure 3. Means \pm SD changes in DDQ and AWQ score in patients with MA abuse under MMT receiving quetiapine and placebo

$P$ value was obtained from the independent $\mathrm{t}$ test; $\mathrm{n}=28$ in each group.

MMT: Methadone Maintenance Treatment; DDQ: Desire for Drug Questionnaire; AWQ: Amphetamine Withdrawal Questionnaire 
explained by the enhanced substance use observed after therapy with typical antipsychotic agents, which block dopamine D2 receptors. Moreover, new antipsychotics, such as quetiapine, show significantly lower dopamine blockade, which cannot result in any elevation in MA use. Quetiapine enables to transiently block the dopamine receptors in the limbic system, which could contribute to a minimal blockade of dopamine in the rewarding process [28]. However, there may also be some other processes that lead quetiapine to reduce MA use. The serotonergic neurotransmission might show one of these processes [29].

We did not evaluate the effects of quetiapine administration on serum and urinary quetiapine. The longer-term intervention may indicate more benefits or side effects of quetiapine. Furthermore, we could not evaluate the pain in individuals with MA abuse. So, it seems that more assessment of pain caused by quitting is needed in further studies.

\section{Conclusions}

We showed that taking quetiapine for 2 months by MA abusers improved withdrawal and craving symptoms. The combination therapy of quetiapine with MMT programs could be introduced to decrease withdrawal syndrome and craving in patients under MMT.

\section{Ethical Considerations}

\section{Compliance with ethical guidelines}

This randomized, double-blinded, placebo-controlled trial was registered on the Iranian website (http://www.irct. ir: IRCT20171106037290N1), the Primary Registry in the World Health Organization (WHO) Registry Network set up in collaboration with the Ministry of Medical and Health Education. The study protocol was approved by the Research Ethics Committee of Kashan University of Medical Sciences (KAUMS). All informed consent forms were reviewed by the Research Ethics Committee of KAUMS.

\section{Funding}

This research was supported by the Clinical Research Development Unit-Matini/Kargarnejad Hospital, Kashan University of Medical Sciences (KAUMS/ 98017).

\section{Author's contributions}

All authors contributed in preparing this article.

\section{Conflict of interest}

The authors declared no conflict of interest.

\section{References}

[1] Noori R, Daneshmand R, Farhoudian A, Ghaderi S, Aryanfard S, Moradi A. Amphetamine-Type stimulants in a group of adults in Tehran, Iran: A rapid situation assessment in twenty-two districts. Iran J Psychiatry Behav Sci. 2016 ; 10(4):e7704. [DOI:10.17795/ijpbs-7704]

[2] Shadloo B, Amin-Esmaeili M, Haft-Baradaran M, Noroozi A, Ghorban-Jahromi R, Rahimi-Movaghar A. Use of amphetamine-type stimulants in the Islamic Republic of Iran, 20042015: A review. East Mediterr Health J. 2017; 23(3):245-56. [DOI:10.26719/2017.23.3.245] [PMID]

[3] Alammehrjerdi Z, Ezard N, Dolan K. Methamphetamine dependence in methadone treatment services in Iran: The first literature review of a new health concern. Asian J Psychiatr. 2018; 31:49-55. [DOI:10.1016/j.ajp.2018.01.001] [PMID]

[4] Tavakoli M, Effatpanah M, Moradi A, Mahjoub A. Methamphetamine dependence among Iranian female methadone patients: A cross-sectional survey of three cities of iran. Iran J Psychiatry Behav Sci. 2018; 12(2):e62866. [DOI:10.5812/ ijpbs.62866]

[5] Dolan KA, Shearer J, MacDonald M, Mattick RP, Hall W, Wodak AD. A randomised controlled trial of methadone maintenance treatment versus wait list control in an Australian prison system. Drug Alcohol Depend. 2003; 72(1):59-65. [DOI:10.1016/S0376-8716(03)00187-X]

[6] Shariatirad S, Maarefvand M, Ekhtiari H. Methamphetamine use and methadone maintenance treatment: An emerging problem in the drug addiction treatment network in Iran. Int J Drug Policy. 2013; 24(6):e115-6. [DOI:10.1016/j.drugpo.2013.05.003] [PMID]

[7] Ghaderi A, Banafshe HR, Mirhosseini N, Motmaen M, Mehrzad F, Bahmani F, et al. The effects of melatonin supplementation on mental health, metabolic and genetic profiles in patients under methadone maintenance treatment. Addict Biol. 2019; 24(4):754-64. [DOI:10.1111/adb.12650] [PMID]

[8] Zorick T, Nestor L, Miotto K, Sugar C, Hellemann G, Scanlon $\mathrm{G}$, et al. Withdrawal symptoms in abstinent methamphetamine-dependent subjects. Addiction. 2010; 105(10):1809-18. [DOI:10.1111/j.1360-0443.2010.03066.x] [PMID] [PMCID]

[9] Ghaderi A, Banafshe HR, Motmaen M, Rasouli-Azad M, Bahmani F, Asemi Z. Clinical trial of the effects of vitamin D supplementation on psychological symptoms and metabolic profiles in maintenance methadone treatment patients. Prog Neuropsychopharmacol Biol Psychiatry. 2017; 79(Pt B):84-9. [DOI:10.1016/j.pnpbp.2017.06.016] [PMID]

[10] Sattar SP, Bhatia SC, Petty F. Potential benefits of quetiapine in the treatment of substance dependence disorders. J Psychiatry Neurosci. 2004; 29(6):452-7. [PMID] [PMCID]

[11] Purdon SE, Malla A, Labelle A, Lit W. Neuropsychological change in patients with schizophrenia after treatment with quetiapine or haloperidol. J Psychiatry Neurosci. 2001; 26(2):137-49. [PMID] [PMCID]

[12] Javdan NS, Ghoreishi FS, Sehat M, Ghaderi A, Banafshe HR. Mental health and cognitive function responses to quetiapine in patients with methamphetamine abuse under methadone maintenance treatment. J Affect Disord. 2019; 251:23541. [DOI:10.1016/j.jad.2019.03.078] [PMID] 
[13] Brown ES, Nejtek VA, Perantie DC, Bobadilla L. Quetiapine in bipolar disorder and cocaine dependence. Bipolar Disord. 2002; 4(6):406-11. [DOI:10.1034/j.1399-5618.2002.02229.x] [PMID]

[14] Telles-Correia D, Barbosa A, Cortez-Pinto H, Campos C, Rocha NB, Machado S. Psychotropic drugs and liver disease: A critical review of pharmacokinetics and liver toxicity. World J Gastrointest Pharmacol Ther. 2017; 8(1):26-38. [DOI:10.4292/wjgpt.v8.i1.26] [PMID] [PMCID]

[15] Ainsworth BE, Haskell WL, Whitt MC, Irwin ML, Swartz AM, Strath SJ, et al. Compendium of physical activities: An update of activity codes and MET intensities. Med Sci Sports Exerc. 2000; 32(Suppl. 9):S498-504. [DOI:10.1097/00005768200009001-00009] [PMID]

[16] Hassani-Abharian P, Mokri A, Ganjgahi H, Oghabian MA, Ekhtiari H. Validation for Persian versions of "Desire for Drug Questionnaire" and "Obsessive Compulsive Drug Use Scale" in Heroin dependents. Arch Iran Med. 2016; 19(9):65965. [DOI:0161909/AIM.0010] [PMID]

[17] Srisurapanont M, Jarusuraisin N, Jittiwutikan J. Amphetamine withdrawal: I. Reliability, validity and factor structure of a measure. Aust N Z J Psychiatry. 1999; 33(1):89-93. [DOI:10.1046/j.1440-1614.1999.00517.x] [PMID]

[18] Khani Y, Nedjat S, Mojtahedzadeh Faghihi M, Mollajan A, Rimaz S. Reliability and validity of the Persian version of Amphetamine Cessation Symptom Assessment (ACSA) questionnaire in Iran. Int J High Risk Behav Addict. 2017; 6(3):e60999. [DOI:10.5812/ijhrba.60999]

[19] Rizkallah E, Stip E, Zhornitsky S, Pampoulova T, Gendron A, Rompre PP, et al. Clinical evolution of substance use disorder patients during treatment with quetiapine: A 12-week, open-label, naturalistic trial. Expert Opin Pharmacother. 2010; 11(18):2947-51. [DOI:10.1517/14656566.2010.524927] [PMID]

[20] Nakama H, Chang L, Cloak C, Jiang C, Alicata D, Haning W. Association between psychiatric symptoms and craving in methamphetamine users. Am J Addict. 2008; 17(5):441-6. [DOI:10.1080/10550490802268462] [PMID] [PMCID]

[21] Buckley PF. Novel antipsychotic medications and the treatment of comorbid substance abuse in schizophrenia. J Subst Abuse Treat. 1998; 15(2):113-6. [DOI:10.1016/S07405472(97)00134-7] [PMID]

[22] Rubio Valladolid G, Casas Brugue M. [Treatment of schizophrenia in subjects with substance use disorders: A review (Spanish)]. Actas Esp Psiquiatr. 2001; 29(2):124-30. [PMID]

[23] Nejtek VA, Avila M, Chen LA, Zielinski T, Djokovic M, Podawiltz A, et al. Do atypical antipsychotics effectively treat co-occurring bipolar disorder and stimulant dependence? A randomized, double-blind trial. J Clin Psychiatry. 2008; 69(8):1257-66. [DOI:10.4088/JCP.v69n0808] [PMID]

[24] Martinotti G, Andreoli S, Di Nicola M, Di Giannantonio M, Sarchiapone M, Janiri L. Quetiapine decreases alcohol consumption, craving, and psychiatric symptoms in dually diagnosed alcoholics. Hum Psychopharmacol. 2008; 23(5):417-24. [DOI:10.1002/hup.944] [PMID]

[25] Tong J, Ross BM, Schmunk GA, Peretti FJ, Kalasinsky KS, Furukawa Y, et al. Decreased striatal dopamine D1 receptor-stimulated adenylyl cyclase activity in human methamphetamine users. Am J Psychiatry. 2003; 160(5):896-903. [DOI:10.1176/appi.ajp.160.5.896] [PMID]
[26] Wang GJ, Smith L, Volkow ND, Telang F, Logan J, Tomasi $\mathrm{D}$, et al. Decreased dopamine activity predicts relapse in methamphetamine abusers. Mol Psychiatry. 2012; 17(9):918-25. [DOI:10.1038/mp.2011.86] [PMID] [PMCID]

[27] Tupala E, Tiihonen J. Dopamine and alcoholism: Neurobiological basis of ethanol abuse. Prog Neuropsychopharmacol Biol Psychiatry. 2004; 28(8):1221-47.[DOI:10.1016/j. pnpbp.2004.06.022] [PMID]

[28] Kapur S, Zipursky R, Jones C, Shammi CS, Remington G, Seeman P. A positron emission tomography study of quetiapine in schizophrenia: A preliminary finding of an antipsychotic effect with only transiently high dopamine D2 receptor occupancy. Arch Gen Psychiatry. 2000; 57(6):553-9. [DOI:10.1001/archpsyc.57.6.553] [PMID]

[29] Tarazi FI, Zhang K, Baldessarini RJ. Long-term effects of olanzapine, risperidone, and quetiapine on serotonin $1 \mathrm{~A}, 2 \mathrm{~A}$ and $2 \mathrm{C}$ receptors in rat forebrain regions. Psychopharmacology (Berl). 2002; 161(3):263-70. [DOI:10.1007/s00213-002-10163] [PMID] 